\title{
MoEDAL, FASER and future experiments targeting dark sector and long-lived particles
}

\author{
Vasiliki A. Mitsou ${ }^{a, *}$ \\ ${ }^{a}$ Instituto de Física Corpuscular (IFIC), CSIC - Universitat de València, \\ C/ Catedrático José Beltrán 2, E-46980 Paterna (Valencia), Spain
}

E-mail: vasiliki.mitsou@ific.uv.es

\begin{abstract}
Theories beyond the Standard Model can predict particles with lifetimes much longer than those of known unstable particles. When produced at high-energy collisions, e.g. at the CERN LHC, these long-lived particles can decay far from the primary interaction vertex. Recently, a number of dedicated experiments have been proposed to search for such objects at the LHC: MoEDALMAPP, MoEDAL-MALL, FASER, CODEX-b, MATHUSLA, AL3X and ANUBIS. The SHiP beam-dump experiment, on the other hand, is planned to operate with the SPS beam to extend the discovery reach of such particles. The already operating MoEDAL detector, its MAPP extension and the planned milliQan experiment are sensitive to particles with exotic electromagnetic charges producing anomalous ionisation. The planned detector design and expected physics sensitivity of these experiments are presented here with emphasis on scenarios explaining the nature of dark matter and the origin of neutrino masses.
\end{abstract}

The Eighth Annual Conference on Large Hadron Collider Physics-LHCP2020

25-30 May, 2020

online

${ }^{*}$ Speaker 


\section{Introduction}

In collider physics there is a growing experimental interest in weakly interacting long-lived particles (LLPs) [1, 2]. These objects travel a macroscopic distance before either decaying within the detector or giving rise to anomalous ionisation. In theory, long lifetimes may be due to small mass splittings, feeble couplings, or a heavy mediator, frequently predicted in models beyond the Standard Model (SM) motivated by either dark matter (DM) or the non-vanishing neutrino masses. For instance, portal-physics models connecting the SM and dark sectors may lead to such LLPs which have a tiny coupling with the SM particles. These models may include dark photons (vector portal), a light scalar (Higgs portal), axion-like particles (pseudoscalar portal), or heavy neutral leptons (fermion portal). Moreover, other theoretical scenarios such as $R$-parity violating supersymmetry may predict the existence of LLPs.

\section{Long-lived neutral particles}

Several new detectors have been proposed to search for LLPs using the LHC beams. The MoEDAL [3] Apparatus for Penetrating Particles (MAPP) [4] has already installed a prototype near the LHCb interaction point (IP) and is analysing Run 2 data. It will comprise two nested subdetectors: the $\mathrm{mQP}$ at the centre and the (larger) LLP around it [5]. The ForwArd Search ExpeRiment (FASER) [6-8] is an approved forward detector situated $~ 480 \mathrm{~m}$ from the ATLAS IP to run in two phases: FASER in Run 3 and FASER2 on the HL-LHC. CODEX-b [9, 10], MATHUSLA [11, 12], AL3X [13], and ANUBIS [14] are proposed experiments located in different angles with respect to the beam axis, featuring various volume sizes and distances from the IP. The COmpact Detector for EXotics at LHCb (CODEX-b) experiment with a nominal fiducial volume of $10 \mathrm{~m} \times 10 \mathrm{~m} \times 10 \mathrm{~m}$ will be situated in a transverse location $\sim 25 \mathrm{~m}$ from the IP. The CODEX- $\beta$ demonstrator is proposed to be operated during Run 3 to provide proof-of-concept [10]. MATHUSLA, the largest in volume and the only surface detector, has constructed a $2.5 \times 2.5 \times 6.5 \mathrm{~m}^{3}$ test stand with eight layers of trackers, which confirmed the background assumptions and gave confidence in projected physics reach [15].

Furthermore, the MoEDAL Apparatus for very Long Lived particles (MALL) [4] is intended to push the search for decays of new charged, massive and extremely long-lived particles, with lifetimes well in excess of a year, by monitoring the MoEDAL trapping volumes [3]. In addition to these LHC projects, the Search for Hidden Particles (SHiP) proposal [16] is a SPS beam-dump experiment for probing LLPs. All these different approaches cover a wide range of parameters such as lifetime, couplings to SM and boost, the latter related to the LLP mass. Moreover, FASER $v$ [17] and the SHiP Scattering and Neutrino Detector (SND) are proposed to detect collider neutrinos.

All these experimental proposals are targeting hidden sectors. For instance in the minimal dark photon model, a new hidden $U(1)$ is added to the SM with a massive gauge field $A_{\mu}^{\prime}$, the dark photon. DM is assumed to be either heavy or contained in a different sector. The dark photon decays visibly into SM states (visible decays). The physics potential of the LLP experiments as a function of the dark photon mass $m_{A^{\prime}}$ and the coupling of dark photon with the standard photon $\epsilon$ is shown in Fig. 1. Comparisons between these experiments with respect to other hidden-sector scenarios are given in Ref. [18] and in Refs. [5, 19] for MAPP-LLP. 


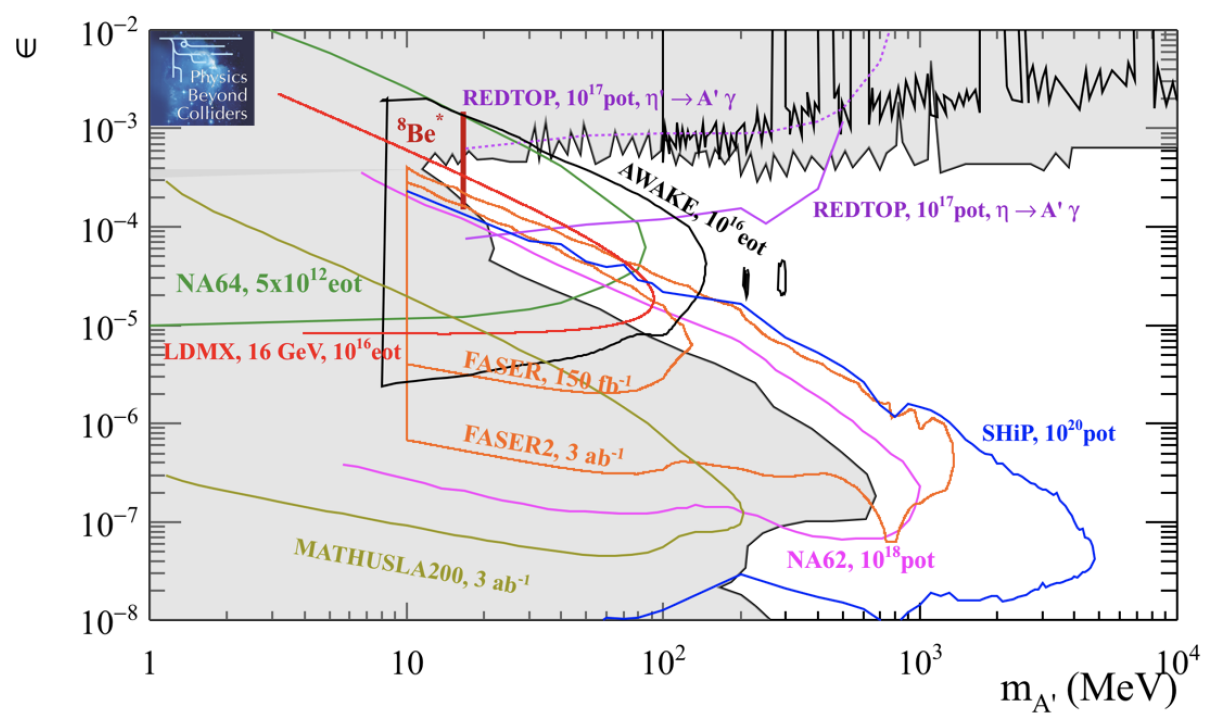

Figure 1: Future upper limits at $90 \% \mathrm{CL}$ for dark photon in visible decays in the plane mixing strength $\epsilon$ versus mass $m_{A^{\prime}}$ for various projects on a $\sim 10-15$ year timescale [18].

The dark Higgs boson case is interesting, in that the dominant production mechanism is through heavy meson decays. A dark Higgs $\phi$ mixes with the SM Higgs with a mixing angle $\theta \ll 1$, leading to exotic $B \rightarrow X_{s} \phi$ decays with $\phi \rightarrow \ell^{+} \ell^{-}$. The respective physics reach for LLP experiments is shown in Fig. 2. The much larger FASER2 detector, to run in HL-LHC, will be needed to cover this scenario [8].

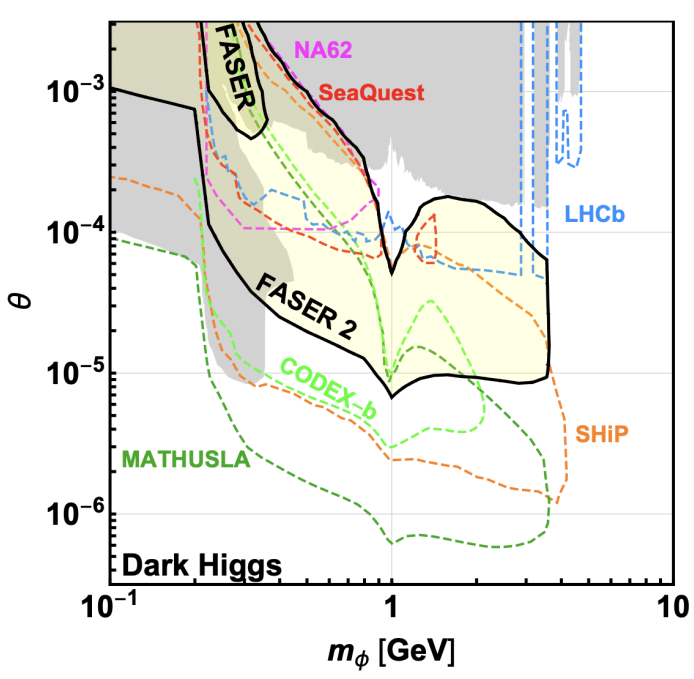

Figure 2: Sensitivity reaches for FASER, FASER2 and other LLP experiments for dark Higgs bosons. The grey-shaded regions are excluded by current bounds [8].

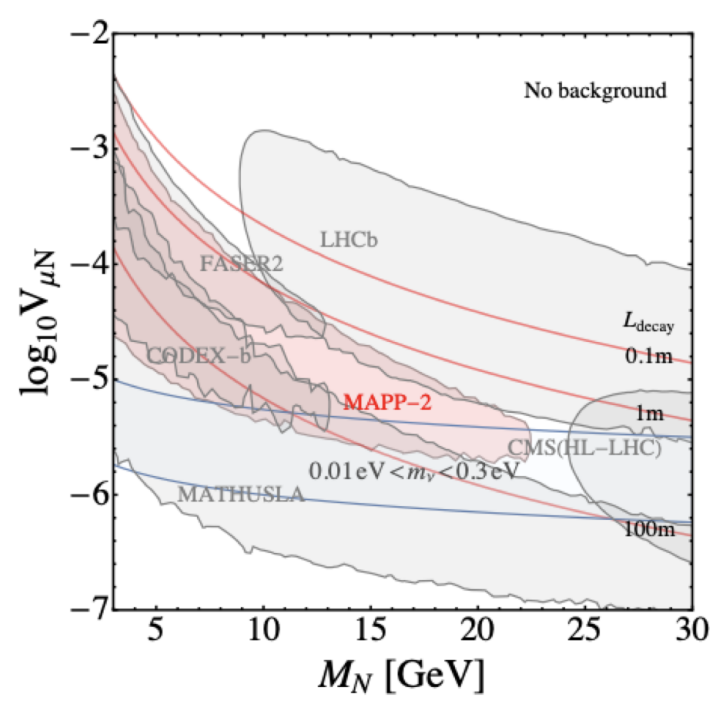

Figure 3: Projected sensitivity of MoEDAL-MAPP and various experiments in the heavy neutrino mass $m_{N}$ and neutrino mixing $V_{\mu N}$ parameter plane for $Z^{\prime}$ production in gauged $B-L$ model [20].

In the fermion portal, right-handed neutrinos $N_{i}$ can be pair produced from the decay of an additional $Z^{\prime}$ boson in the gauged $B-L$ model, which also contains a singlet Higgs $\chi$ that 
spontaneously breaks the extra $U(1)_{B-L}$ gauge symmetry. From Fig. 3, it is evident that MAPP-2, due to take data in HL-LHC, will fill the gap left by CMS, LHCb, MATHUSLA, FASER2 and CODEX-b [20]. Numerous studies have compared the physics reach of LLP experiments for different scenarios of heavy neutral leptons [18, 21-23].

$R$-parity violating supersymmetry also predicts LLPs, e.g. light long-lived neutralinos $\tilde{\chi}_{1}^{0}$ decaying via $\lambda_{i j k}^{\prime}$ couplings to charged particles. Benchmark scenarios related to either charm or bottom mesons decaying into the light neutralino have been considered showing that the LLP detectors can cover various $\tilde{\chi}_{1}^{0}$ lifetimes $[22,24,25]$.

\section{Stable milli-charged particles}

Milli-charged particles ( $\mathrm{mCP}$ ) have been discussed in connection with the mechanism of electric charge quantisation and possible non-conservation of electric charge. There are two experiments planned to run at the LHC that are sensitive to the detection of the low ionisation coming from an mCP. The milliQan detector $[26,27]$ to be installed near the CMS IP and the MAPP-mQP subdetector near the LHCb/MoEDAL cavern [4].

A well-motivated mechanism to predict such mCPs is to introduce a new $U(1)$ in dark sector with a massless dark photon $A^{\prime}$ and a massive dark fermion $\psi$ with charge much less than that of en electron as a result of kinetic mixing. MAPP-mQP and milliQan expect to achieve a low-mass $\left(m_{\psi} \simeq 0.1-1 \mathrm{GeV}\right)$ sensitivity to dark-fermion mCPs with $Q=3 \times 10^{-3} e$ [5, 27]. Furthermore, it has been shown that MAPP-mQP can detect a heavy neutrino with a large enough electric dipole moment considered to be a member of a fourth generation lepton doublet [28].

A milliQan 1\% scale demonstrator was installed in the tunnel above CMS and a $13 \mathrm{TeV}$ search excluded mCP masses of $20-4700 \mathrm{MeV}$ for charges varying between $0.006 e$ and $0.3 e$ depending on mass [29]. Data taken from a MAPP-mQP prototype during Run 2 are currently being analysed [4] and the full subdetector is expected to be fully operational during Run 3.

\section{Summary and outlook}

There is an ever increasing interest in long-lived particle searches at the LHC (and not only) including the dark sector and the neutrino masses [1, 18]. Besides the efforts in the generalpurpose experiments ATLAS and CMS, additional complementary experiments have been approved (MoEDAL-MAPP, FASER) aiming at start data-taking in Run 3 or have been proposed (MATHUSLA, CODEX-b, milliQan, ANUBIS, AL3X). In addition to these LHC detectors, the SHiP experiment at the SPS is also planned to explore hidden sectors. Several of these experiments have constructed/taken data or plan to build small-scale demonstrators to measure backgrounds and provide proof-of-concept. A different perspective - the long lifetime - on the dark sector is expected in searches in the LHC Run 3 and beyond.

\section{Acknowledgments}

This work was supported in part by the Generalitat Valenciana via a special grant for MoEDAL and via the Project PROMETEO-II/2017/033, and by the Spanish MICIU / AEI and the European Union / FEDER via the grant PGC2018-094856-B-I00. 


\section{References}

[1] J. Alimena et al., J. Phys. G 47 (2020) 090501 [1903. 04497].

[2] L. Lee, C. Ohm, A. Soffer and T.-T. Yu, Prog. Part. Nucl. Phys. 106 (2019) 210 [1810.12602].

[3] MoEDAL collaboration, Int. J. Mod. Phys. A29 (2014) 1430050 [1405 . 7662].

[4] J. L. Pinfold, Phil. Trans. Roy. Soc. Lond. A377 (2019) 20190382.

[5] M. Staelens, in Meeting of the Division of Particles and Fields of the American Physical Society, 10, 2019, 1910.05772.

[6] J. L. Feng, I. Galon, F. Kling and S. Trojanowski, Phys. Rev. D 97 (2018) 035001 [1708.09389].

[7] FASER collaboration, Phys. Rev. D 99 (2019) 095011 [1811 . 12522].

[8] FASER collaboration, 1901.04468.

[9] V. V. Gligorov, S. Knapen, M. Papucci and D. J. Robinson, Phys. Rev. D 97 (2018) 015023 [1708.09395].

[10] G. Aielli et al., 1911.00481.

[11] MATHUSLA collaboration, JINST 15 (2020) C06026 [1901.04040].

[12] MATHUSLA collaboration, 2009.01693.

[13] V. V. Gligorov, S. Knapen, B. Nachman, M. Papucci and D. J. Robinson, Phys. Rev. D 99 (2019) 015023 [1810.03636].

[14] M. Bauer, O. Brandt, L. Lee and C. Ohm, 1909. 13022.

[15] M. Alidra et al., 2005. 02018.

[16] SHIP collaboration, JINST 14 (2019) P03025 [1810.06880].

[17] FASER collaboration, Eur. Phys. J. C 80 (2020) 61 [1908. 02310].

[18] J. Beacham et al., J. Phys. G 47 (2020) 010501 [1901.09966].

[19] V. A. Mitsou, PoS CORFU2019 (2020) 009.

[20] F. Deppisch, S. Kulkarni and W. Liu, Phys. Rev. D 100 (2019) 035005 [1905. 11889].

[21] F. Kling and S. Trojanowski, Phys. Rev. D 97 (2018) 095016 [1801. 08947].

[22] D. Dercks, H. K. Dreiner, M. Hirsch and Z. S. Wang, Phys. Rev. D 99 (2019) 055020 [1811.01995].

[23] M. Hirsch and Z. S. Wang, Phys. Rev. D 101 (2020) 055034 [2001.04750].

[24] D. Dercks, J. De Vries, H. K. Dreiner and Z. S. Wang, Phys. Rev. D 99 (2019) 055039 [1810.03617].

[25] H. K. Dreiner, J. Y. Günther and Z. S. Wang, 2008. 07539.

[26] A. Haas, C. S. Hill, E. Izaguirre and I. Yavin, Phys. Lett. B 746 (2015) 117 [1410 . 6816].

[27] A. Ball et al., 1607.04669.

[28] M. Frank, M. de Montigny, P.-P. A. Ouimet, J. Pinfold, A. Shaa and M. Staelens, Phys. Lett. B 802 (2020) 135204 [1909.05216].

[29] A. Ball et al., Phys. Rev. D 102 (2020) 032002 [2005.06518]. 